\title{
Géolinguistique
}

15 | 2015

La géographie linguistique au Brésil

\section{L'EALMG (1977) et l'ALMS (2007) : que dévoilent ces atlas sur la méthodologie et l'enregistrement de la norme lexicale du portugais du Brésil ?}

EALMG (1977) and ALMS (2007): What Do these Atlases Reveal about the Methodology and Registration of the Brazilian Portuguese Lexical Standard?

\section{Aparecida Negri Isquerdo}

Traducteur : Aline Saddi Chaves

\section{(2) OpenEdition}

\section{Journals}

Édition électronique

URL : http://journals.openedition.org/geolinguistique/606

DOI : $10.4000 /$ geolinguistique.606

ISSN : 2650-8176

Éditeur

UGA Éditions/Université Grenoble Alpes

Édition imprimée

Date de publication : 1 décembre 2015

Pagination : 39-66

ISBN : 978-2-84310-317-9

ISSN : 0761-9081

Référence électronique

Aparecida Negri Isquerdo, "L'EALMG (1977) et l'ALMS (2007) : que dévoilent ces atlas sur la

méthodologie et l'enregistrement de la norme lexicale du portugais du Brésil ? », Géolinguistique [En ligne], 15 | 2015, mis en ligne le 15 février 2019, consulté le 05 novembre 2020. URL : http:// journals.openedition.org/geolinguistique/606; DOI : https://doi.org/10.4000/geolinguistique.606 


\title{
L'EALMG (1977) et l'ALMS (2007) : que dévoilent ces atlas sur la méthodologie et l'enregistrement de la norme lexicale du portugais du Brésil ?'
}

\author{
Aparecida Negri Isquerdo \\ Universidade Federal de Mato Grosso do Sul - CNPq (Brésil)
}

\section{Résumé}

Ce travail analyse deux atlas linguistiques régionaux publiés au Brésil sur un laps de temps de 30 ans : l'Ébauche d'un Atlas linguistique de Minas Gerais - EALMG (Ribeiro, Zágari, Passini \& Gaio, 1977) et l'Atlas linguistique de Mato Grosso do Sul - ALMS (Oliveira, 2007) qui couvrent, respectivement, les États de Minas Gerais (région Sud-Est du Brésil) et Mato Grosso do Sul (Centre-Ouest brésilien). Ce travail a analysé en premier les données cartographiées dans l'EALMG, en fonction de l'époque de peuplement de l'État de Minas Gerais (XVII ${ }^{\mathrm{e}}$ et $\mathrm{XVIII}^{\mathrm{e}}$ siècles), de même que les autochtones de Minas Gerais (nommés Mineiros), ainsi que les Paulistas et Paranaenses (comme sont désignés les habitants des États de São Paulo et Paraná) ont été les colonisateurs du sud de l'ancien État de Mato Grosso ( $\mathrm{XIX}^{\mathrm{e}}$ et $\mathrm{XX}^{\mathrm{e}}$ siècles). Cette recherche vise donc à réaliser une étude comparative entre les données cartographiées de l'EALMG et de l'ALMS en ce qui concerne le phénomène signalé. Ainsi, l'objectif est d'identifier la distribution spatiale des noms pour l'arc-en-ciel, et aussi de vérifier le déroulement de dissémination et de fixation de ces désignations, le niveau de résistance de certains termes lexicaux selon leur espace géographique et l'histoire sociale de la région étudiée.

1. Article traduit du portugais par Aline Saddi Chaves. 


\title{
Mots-clés
}

Atlas linguistique, EALMG, ALMS, lexique, histoire sociale.

\begin{abstract}
This paper intends to analyze two state linguistic atlas published in Brazil in a 30-year interregnum, Minas Gerais Linguistic Atlas Draft - EALMG (Ribeiro, Zágari, Passini \& Gaio, 1977) and Mato Grosso do Sul Linguistic Atlas - ALMS (Oliveira, 2007) which cover Minas Gerais (Southeastern part of Brazil) and Mato Grosso do Sul (Brazilian Midwest) states, respectively. The text is structured in two parts. The data mapped by EALMG are taken as a starting point, considering the population process of Minas Gerais state (17th-18th centuries) and also the fact that the south of Mato Grosso state was colonized by people from Minas Gerais, São Paulo and Paraná (19th and 20th centuries). Thus, this is an attempt to carry out a comparative study involving the data mapped by EALMG and ALMS, related to the selected phenomenon, aiming at identifying the special distribution of names of "rainbow", as well as verifying dissemination and fixation processes of these designations-bigger or smaller resistance of certain lexical items, according to the geographical space and the social story of the region where the research was conducted.
\end{abstract}

\section{Keywords}

Linguistic Atlas, EALMG, ALMS, lexicon, social history.

Depuis les dernières décennies, les recherches en linguistique au Brésil connaissent une large diffusion en ce qui concerne les études dialectales dans une perspective géolinguistique, en grande partie motivées par la publication du projet Atlas linguistique au Brésil (1996). Ce dernier a en effet inauguré une nouvelle période dans les recherches dialectologiques et géolinguistiques dans le pays, du fait d'avoir fourni à la communauté académique, notamment, une méthodologie basée sur les orientations contemporaines pour les recherches dans le domaine. Ouvrage de grande portée, cet atlas invite en effet à une nouvelle démarche méthodologique "pelo tratamento pluridimensional dado aos fatos geolinguísticos ${ }^{2} »$ (Mota \& Cardoso, 2006, p. 24). L'ALiB se donne pour but principal la description de la langue portugaise parlée dans les 26 unités de la fédération du Brésil,

2. «de par son traitement pluridimensionnel donné aux faits géolinguistiques ». 
à partir d'enquêtes menées auprès de 1100 sujets parlants le portugais, originaires des 250 localités qui intègrent le réseau de points de l'ALiB.

Le présent article est organisé autour de la présentation et de la discussion des principes méthodologiques de deux atlas linguistiques recouvrant, respectivement, les États brésiliens de Minas Gerais et Mato Grosso do Sul. Trois décennies les séparent : 1977 (Esboço de um Atlas Lingüístico de Minas Gerais - EALMG) et 2007 (Atlas Lingüístico de Mato Grosso do Sul-ALMS). La date de parution des ouvrages constitue en soi un indicateur de la démarche théorique adoptée par leurs auteurs. Alors que l'EALMG se tourne vers la dialectologie intéressée à l'étude de la variation diatopique, plus précisément l'interprétation des faits linguistiques dans des aires majoritairement rurales, l'ALMS de son côté a pour objet aussi bien les dialectes ruraux que les dialectes sociaux et urbains. En plus de sa dimension diatopique, ce dernier prend en compte les variations diastratiques, diasexuelles et diagénérationnelles.

Notre démarche ici est double : i) présenter et mener une réflexion sur la méthodologie qui se trouve à l'origine de la construction de chacun des atlas, en établissant un parallèle entre les deux ouvrages en ce qui concerne leurs principes théoriques propres - à savoir la dialectologie et la géolinguistique - et, par la suite, les paramètres méthodologiques qui les rendent singuliers; ii) à partir des cartes communes aux deux atlas, nous abordons les différentes désignations attribuées au phénomène atmosphérique communément appelé arc-en-ciel (bande lumineuse aux rayons colorés et courbées qui peuvent apparaître dans le ciel à la suite d'une pluie). La démarche adoptée consiste à faire une étude croisée des données cartographiées par l'EALMG et l'ALMS pour le phénomène en question, en vue d'identifier, d'une part, la distribution spatiale des noms d'arc-en-ciel et de vérifier, d'autre part, les processus de diffusion et de figement de ces désignations - le degré plus ou moins élevé de résistance de certains items lexicaux selon l'espace géographique et l'histoire sociale de la région étudiée.

\section{Contextualisation géographique}

Les deux atlas étudiés documentent le parler de deux États brésiliens appartenant à deux grandes régions du pays, distinctes non seulement du point de vue géographique mais aussi et surtout en termes d'histoire sociale.

L'EALMG présente des données de la langue parlée dans le Minas Gerais, un État qui intègre la région Sud-Est du Brésil et dont les premières vagues de peuplement datent de la fin du XVII ${ }^{\mathrm{e}}$ siècle. À l'origine de ce peuplement se trouve le mouvement des explorateurs dits bandeirantes, 
lesquels avancèrent vers l'intérieur de la colonie à la recherche de mines d'or et de pierres précieuses à des fins d'exploitation. Il s'ensuivit l'apparition d'agglomérations à l'intérieur du pays.

L'ALMS documente des données linguistiques fournies par des sujets parlants de la langue portugaise de Mato Grosso do Sul, une unité administrative située dans la région Centre-Ouest du Brésil, la dernière en date à avoir été peuplée par le processus de colonisation du territoire brésilien, à partir du $\mathrm{Xx}^{\mathrm{e}}$ siècle, à l'occasion du programme «Marche vers l'Ouest», inauguré en 1937 par le président Getúlio Vargas, suite à la politique de promotion de la migration (création de colonies agricoles, construction de routes, programmes de réforme agraire...) en vue du peuplement du Brésil central et de l'Amazonie.

\section{Présentation des atlas}

\section{1. Ébauche d'un Atlas Linguístico de Minas Gerais - EALMG : méthodologie}

L'EALMG a été conçu par Mário Roberto Lobuglio Zágari, José Ribeiro, José Passini et Antônio Gaio, enseignants chercheurs attachés à l’Université fédérale de Juiz de Fora. Le premier volume paraît en 1977 : il s'agit du deuxième atlas linguistique élaboré au Brésil. À l'origine, ce projet prévoyait une publication en quatre volumes, destinés à faire :

[...] inventário (volumes I, II e III) e da sistematização e interpretação (volume IV) das variantes do português de Minas Gerais, por parte do falante de camadas menos cultas. Exame da norma de uso da região na fonética, fonologia, lexicologia e morfossintaxe ${ }^{3}$. (Ribeiro et al., 1977, p. 18)

Le volume I est «na verdade, o único volume publicado, uma vez que os demais, previstos no plano inicial da obra, devido a percalços diversos, não chegaram a concretizar-se ${ }^{4} \gg$ (Rocha, 2012, p. 81).

\subsubsection{Le réseau de points}

Le réseau de l'EALMG comprend 116 localités, distribuées sur environ $600000 \mathrm{~km}^{2}$, avec aussi bien de grands centres urbains, l'accent étant mis

3. «[...] l'inventaire (volumes I, II et III) et la systématisation et interprétation (volume IV) des variantes du portugais de Minas Gerais employées par les sujets parlants appartenant aux couches moins cultivées. Examen de la norme d'usage de la région en ce qui concerne la phonétique, la phonologie, la lexicologie et la morphosyntaxe.»

4. «en réalité, le seul volume publié, étant donné que les autres, prévus dans le projet initial de l'ouvrage, n'ont pas pu être achevés pour diverses contraintes». 
sur les aspects économique, industriel, touristique et historique, que des villages d'une dizaine de maisons :

Buscou-se atingir tanto os centros tradicionais do estado como as cidades industrial e comercialmente mais importantes, ou ainda aquelas de comprovada história pelo passado colonial; enfim os centros de irradiação, $e$ também os pontos marginais, o fim-da-estrada, o ermo de menos de 1.000 habitantes, afastado do asfalto e sem outra via de comunicação que não o retorno à sede do município ${ }^{5}$. (Ribeiro et al., 1977, p. 29)

Le choix des localités a été réalisé en trois étapes et sur la base de critères distincts. Tout d'abord ont été sélectionnés, à travers le critère historico-démographique, les cinquante premiers points. La recherche dans cet ensemble de localités étant conclue, les premières cartes étant confectionnées, l'équipe a émis des hypothèses phonétiques et lexicologiques pour la sélection des cinquante et un autres points, cette fois sur la base de critères linguistiques : "rastreio de determinados fatos fonéticos ou acompanhando o uso desse ou daquele vocábulo ${ }^{6}$ (Ribeiro et al., 1977, p. 29). Enfin, dans la troisième étape ont été choisies les quinze localités restantes qui «surgiram exatamente onde uma dúvida ou outra persistia ${ }^{7}$ (ibid.).

Il convient de noter que la méthodologie mise en place a assuré la représentativité des localités aux traits distincts, sachant que sur le tableau des 116 localités qui forment le réseau de points de l'EALMG figurent : «a) os grandes centros (universidade, arcebispado); b) os centros médios (ensino médio, bispado); c) os pequenos (escola, paróquia); d) os vilarejos ( simples escolas rurais $)^{8} \gg$ (ibid.).

Outre la liste de points, l'atlas fournit aux lecteurs des données historiques générales sur la formation de l'État de Minas Gerais, aussi bien sur la construction des premières routes ( $\mathrm{XVI}^{\mathrm{e}}$ siècle), l'apparition des premiers villages et les différentes périodes économiques, que sur le panorama général de l'État en ce qui concerne sa population, son économie et le transport dans les années 1970. Par la suite, l'atlas présente des données

5. «On a essayé d'atteindre aussi bien les centres traditionnels de l'État que les villes industriellement et commercialement plus importantes, voire celles dont l'histoire est marquée par le passé colonial; enfin les centres de rayonnement mais aussi les points marginaux, les fins fonds, les hameaux de moins de mille habitants, éloignés de la route et sans autre voie de communication que le retour au siège de la municipalité.»

6. «dépistage de certains faits phonétiques ou l'emploi de tel ou tel vocable».

7. «apparues exactement là où un doute ou l'autre persistait».

8. «a) les grands centres (université, archevêché); b) les centres moyens (collègelycée, évêché); c) les petits centres (école, paroisse); d) les villages (simples écoles rurales)». 
historiques sur chacune des localités étudiées, à savoir des informations toponymiques (origine du nom de la localité et les noms précédents), des ethnonymes, le numéro de la localité sur le réseau de points de l'atlas, des données topographiques (altitude), la localisation dans l'État (zone, microrégion), des coordonnées géographiques, la population, les moyens de transport et les principales activités économiques.

\subsubsection{Le profil des informateurs}

Pour l'élaboration de l'EALMG, les informateurs ont été choisis selon les critères suivants : homme ou femme; âge compris entre 30 et 50 ans; analphabète ou scolarisé(e) jusqu'au CM2 et/ou ayant suivi récemment des cours d'alphabétisation fonctionnelle $\left(\mathrm{MOBRAL}^{9}\right)$; issu(e) de la localité concernée, y compris sa famille, elle aussi née et élevée dans la même localité; si marié(e), le/la conjoint(e) devait appartenir à la même localité; profession variable; ne pas avoir fait beaucoup de déplacements et ne pas être né(e) dans une localité autre que celle de sa résidence; ne pas avoir effectué le service militaire et faire preuve de bonnes conditions de santé et d'une bonne prononciation. Sur l'atlas figurent les données des informateurs des cinquante premières localités observées : dans 27 d'entre elles, l'enquête a été menée avec un seul informateur, alors que dans 23 autres il y a eu la participation de plus d'un informateur - un principal et le(s) auxiliaire(s), ces derniers entre un et cinq par localité. Sur le montant total des informateurs dont les données figurent dans l'atlas, trois seulement étaient des femmes : une principale (31 ans - Araxá), une auxiliaire (33 ans Caxambu), un informateur unique (35 ans - Ponte Nova). On voit ainsi que seulement deux des informateurs étaient du sexe féminin, ce qui confirme que l'EALMG est un atlas conçu selon la tradition de la dialectologie, laquelle a toujours privilégié les informateurs du type HARAS (homme, adulte, rural, analphabète et sédentaire) (Zágari, 1998, p. 36). Le projet a toutefois avancé lorsque, dans la mesure du possible, les enquêtes ont été menées avec plus d'un informateur, aux âges approximatifs, un principal et un auxiliaire. Cette démarche méthodologique avait pour but de confirmer et d'enrichir les données. Elle est adoptée à l'heure actuelle par Thun (1998) et ses adeptes dans des projets d'atlas linguistiques, en particulier ceux à visée topodynamique, en vue d'élargir l'éventail des informations obtenues et de confirmer les variétés linguistiques documentées dans des territoires fortement marqués par les contacts linguistiques.

9. Promulgué en 1967, le Mouvement brésilien d'alphabétisation (MOBRAL) était un projet du gouvernement brésilien destiné à l'alphabétisation fonctionnelle des jeunes et adultes n'ayant pas reçu de formation scolaire. 


\subsubsection{Le questionnaire linguistique et les enquêtes}

Le projet qui se trouvait à l'origine de l'EALMG prévoyait deux types de démarches pour le recueil des données : l'approche directe et l'approche indirecte.

Selon Ribeiro et al., «fez-se a pesquisa direta, tão espontânea quanto possível, in loco, em 116 municípios mineiros, através de uma conversação dirigida, mediante questionário previamente preparado para tal fim ${ }^{10}{ }$ (1977, p. 25). Les auteurs expliquent aussi (p. 25) que la conception du questionnaire linguistique a suivi les orientations consignées dans l'ouvrage d'Antenor Nascentes (1958), Bases para a elaboração do Atlas Linguístico do Brasil; sur celui de Serafim da Silva Neto (1975), Guia para Estudos Dialetológicos, et sur les cartes de l'APFB - Atlas Prévio dos Falares Baianos (Rossi, 1963). Le questionnaire regroupe 415 questions, rassemblées dans huit champs sémantiques. Les enquêtes ont été menées par six enquêteurs, parmi lesquels figurent quatre auteurs de l'atlas.

La recherche indirecte s'est mise en place après la réalisation des cinquante premiers enregistrements «quando já se esboçavam os primeiros limites virtuais -isófonas e isoléxicas - [...] com a finalidade de comprovar, no domínio do léxico, a validade ou não da isoléxica 'traçada', na precisão do seu limite ${ }^{11} \gg$ (Ribeiro et al., 1977, p. 30). Il s'agissait, à cette étape de la recherche, d'envoyer une lettre au professeur de portugais de la localité (à défaut, la directrice de l'école publique du niveau primaire), toujours à travers la mairie locale. La lettre contenait les consignes, les fiches de la localité et de l'informateur, ainsi que le questionnaire dirigé aux données visées (ibid., p. 31). Ces questionnaires ont été envoyés à 672 municipalités, dans le but exclusif «de testar e controlar as respostas obtidas em determinadas áreas ${ }^{12}{ }$ (ibid., p. 18). Parmi les informateurs, 409 ont répondu aux questions, mais 107 n'ont pas été pris en compte par manque de l'une des conditions exigées (âge, scolarité, lieu de naissance ou de résidence de l'informateur). Finalement, seules 302 enquêtes ont été réalisées par correspondance.

Le projet original de l'EALMG ne se limitait pas à la récolte des données géolinguistiques, car il prévoyait en parallèle une enquête sociolin-

10. «la recherche a été de type direct, aussi spontanée que possible, in loco, dans 116 villes de Minas Gerais, à travers un entretien dirigé basé sur un questionnaire préalablement conçu à cette fin».

11. «quand les premières limites virtuelles - isophones et isolexicales - s'esquissaient [...] dans le but de prouver, dans le domaine du lexique, la validité ou non de l'isolexique tracé, dans la précision de sa limite».

12. «de tester et de contrôler les réponses obtenues dans certains domaines». 
guistique, de manière à approfondir la récolte des données en trois ou quatre points,

[...] quer na tentativa de se descobrir até que ponto antigos quilombos ou negros alforriados influenciaram a fala de certos locais, ou que tipo de linguajar tais negros conservaram, quer para saber se há ou não um pidgin na fala do «branco» ao se comunicar com agrupamentos indígenas de algumas regiões. Esses pontos deveriam ter figurado no volume III do Atlas que, como já assinalado, não chegou a ser publicado ${ }^{13}$. (Ribeiro et al., 1977, p. 333-34)

\subsubsection{L'organisation du Esboço de um Atlas Linguístico de Minas Gerais}

Le deuxième atlas linguistique publié au Brésil a été réalisé par José Ribeiro, Mário Roberto Lobuglio Zágari, José Passini et Antonio Gaio. Quoique conçu pour être publié en quatre volumes, un seul volume a été achevé, la probabilité de publication des trois autres étant presque nulle, pour des raisons diverses qui ont empêché l'équipe responsable de conclure le travail (décès ou départ à la retraite de quelques auteurs, inachèvement du travail de la part de l'autre équipe responsable, s'ajoutant à tout cela des problèmes liés au financement de la publication des autres volumes).

D'après l'un des derniers auteurs en vie de cet atlas (Mário Roberto Zágari), les trois volumes restants étaient pratiquement prêts. Le volume I fournit, après la «Table des matières» et la «Présentation », le «Plan» complet de l'ouvrage avec des détails sur le contenu prévu pour chaque volume. Apparaissent ensuite le «Résumé» de la publication et l' «Introduction», suivis du premier chapitre «Méthodologie» qui présente en détails le parcours des différentes étapes de la recherche, en plus de la justification des procédures méthodologiques adoptées par l'équipe responsable de l'atlas dans ses différentes étapes d'exécution (contrôle des variables, questionnaire linguistique, enquête directe et indirecte, procédures adoptées dans l'enquête, dans la sélection des informateurs, les enquêteurs, les enregistrements des enquêtes, la transcription phonétique, la présentation des cartes).

13. «[...] que ce soit dans le but de chercher à découvrir jusqu'à quel point les anciennes communautés quilombo de Noirs affranchis auraient influencé le parler de certaines régions, ou quel type de dialecte aurait été conservé par ces Noirs, mais aussi pour savoir s'il existe ou non un pidgin dans le parler du Blanc lorsque ce dernier communique avec des regroupements indigènes installés dans certaines régions. Ces points auraient dû apparaître dans le volume III de l'atlas qui, comme nous l'avons signalé, n'a pas été publié.» 
Le deuxième chapitre porte sur les «Localités» observées. Outre la liste des 116 points qui forment le réseau, ce chapitre présente des données historiques générales sur les différentes régions de l'État de Minas Gerais et des informations spécifiques sur les 50 premiers points où le recueil des données linguistiques a eu lieu.

La publication présente ensuite les «Annexes» avec deux sections. La première section, intitulée «L'enquête par correspondance», contient les instructions fournies pour cette modalité de recueil de données (section 1.1); la fiche de la localité (section 1.2); la fiche de l'informateur (section 1.3); et le type de questionnaire utilisé dans l'enquête indirecte (section 1.4). La deuxième section est consacrée aux «Informateurs» et se divise en deux parties : la section 2.1 contient la présentation du modèle de la fiche de l'informateur utilisée par l'EALMG, et la section 2.2 fournit des précisions sur les informateurs (appartenant eux aussi aux 50 premières localités du réseau), distribués selon les points et dûment identifiés en tant qu'informateur principal, auxiliaire ou unique.

Le troisième chapitre, intitulé «Cartes», présente l'atlas proprement dit et réunit quatre cartes introductives (non numérotées) : i) Minas Gerais au Brésil; ii) Principaux accidents géographiques de Minas Gerais; iii) Les zones de Minas; iv) Les localités (cette carte porte l'identification des points du réseau selon les trois étapes de la récolte des données : noir $\rightarrow$ les 50 premiers points; rose $\rightarrow$ les 51 points suivants; bleu $\rightarrow$ les 15 derniers points); v) Les enquêteurs, identifiés également par des couleurs différentes.

Dans la suite du chapitre sont présentées les 73 cartes linguistiques ainsi distribuées : 45 cartes onomasiologiques recouvrant des données lexicales ou lexico-phonétiques, selon les aires sémantiques temps et fêtes populaires enfantines de rue, en plus des 3 cartes d'isophones et les 25 cartes isolexicales des phénomènes mis en évidence par les cartes onomasiologiques. Étant donné que l'EALMG n'a pas réalisé le contrôle systématique des variables sociales, la représentation du phénomène dans la localité sur les cartes lexicales et phonétiques a suivi la démarche suivante, selon Ribeiro et al. : «cores vermelho, azul, verde, preto, amarelo e marrom, em círculos cheios e vazios ${ }^{14} \gg(1977$, p. 32). Quant aux cartes isophones et isolexicales, la ligne continue (-) indique «as regiões cujos falantes, em seu vocabulário ativo, ou em sua cadeia sonora, só empregam aquele vocábulo ou segmento ${ }^{15}{ }$ (ibid.); la ligne interrompue (- - ) «demonstra

14. «les couleurs rouge, bleu, vert, noir, jaune et marron en cercles pleins et vides».

15. «les régions où les sujets parlants, dans leur vocabulaire actif ou leur chaîne sonore, n'emploient que le vocable du segment». 
a existência, na área, de variantes da forma dada ${ }^{16}{ }$ (ibid.), alors que la ligne interrompue par des points (-•-•-) indique l'espace «onde a correspondência demonstrou a existência do vocábulo ainda não confirmado pelas pesquisas in $l^{\circ}{ }^{17}{ }^{17}$ (ibid.).

Après la présentation des cartes, l'EALMG fournit au lecteur un «Glossaire» (chapitre 4) qui rassemble 139 entrées composées de variantes documentées dans les cartes de l'atlas. Les entrées du glossaire sont ainsi organisées : l'entrée (variante en question), en lettres capitales et caractères gras, accompagnée du symbole * ou ** qui indiquent, respectivement, si la variante figure dans les dictionnaires dans une autre acception ou si elle n'a pas été relevée. Pour ce faire, deux dictionnaires de la langue portugaise ont servi de base : Novo Dicionário da Língua Portuguesa, de Aurélio Buarque de Hollanda Ferreira (1975) et le Dicionário Contemporâneo da Lingua Portuguesa, de Caldas Aulete (1964). L'entrée indique aussi la classe grammaticale de la variante lexicale, puis, en italique, son équivalent dans le lexique de la langue portugaise des sujets parlants de Minas Gerais enquêtés pour l'EALMG (définition synonymique).

Voici des exemples d'entrées du glossaire :

- CHUVA-DE-FLOR**, s.f. : chuva-de-pedra ${ }^{18}$;

- INVERNO*, s.m. : garoa $^{19}$;

- ZELAÇÃO, s.f. : estrela cadente ${ }^{20}$.

(Ribeiro et al., 1977, p. 239, 240, 241)

Le glossaire contient seulement trois entrées dont la définition n'est pas de type synonymique :

- BOCA-DE-FORNO, s.f. : o mesmo que chicotinho-queimado, no NE de Minas. (Dicionarizado de maneira vaga) ${ }^{21}$;

- BÚRICA*, s.f. : buraquinho na terra aonde caem as bolinhas no jogo de gude ${ }^{22}$;

16. «démontre l'existence, dans l'aire, de variantes de la forme donnée ».

17. «où la correspondance a démontré l'existence du vocable encore non confirmé par les recherches in loco».

18. Pluie-de-fleur, $\mathrm{n}$. f. : pluie-de-pierre.

19. Hiver, n. m. : bruine.

20. Météore, n. f. : étoile filante.

21. Jeu d'enfant traditionnel, identique au jeu de corde dit chicotinho-queimado, au nord-est de Minas Gerais (entrée dans le dictionnaire de manière imprécise).

22. Petit trou dans la terre où convergent les billes dans le jeu de billes. 
— TROMBA-D’ÁGUA*, s.f. : chuva muito forte que, embora rápida, produz enchentes ${ }^{23}$.

(Ribeiro et al., 1977, p. 239, 241)

Le volume I de l'EALMG se termine par une «Bibliographie» avec les travaux mentionnés dans l'élaboration de l'atlas.

Pour conclure cette partie de commentaires sur l'EALMG, il convient d'expliquer que même si les trois volumes prévus n'ont pas été publiés, leurs données ont été recueillies tel qu'il était prévu dans le projet initial de l'ouvrage, et font actuellement l'objet d'études ponctuelles de la part des membres de l'équipe de recherche, y compris des étudiants qu'ils dirigent. L'étude de Zágari, par exemple, trace le bilan de la recherche réalisée après l'élaboration du volume I de l'atlas, en se référant aux orientations de la sociolinguistique labovienne : «[...] levantamento direto, in loco, em diferentes localidades das Gerais, precisamente 184 (cento e oitenta e quatro) pontos através de uma conversação semi-dirigida, mediante questionários específicos preparados para tal finalidade ${ }^{24} \gg(1998$, p. 31).

Selon ce chercheur, après dix ans de recherche et plus de six mille heures d'enregistrement, il a été possible d'arriver à la conclusion suivante :

Minas Gerais apresenta acentos, fones, ritmos de fala, e preferências lexicais distintas em, pelo menos, três de suas regiões independentemente de seus estratos sociais. Há um falar no sul e no Triângulo que se distingue do Norte, os quais, por sinal, se diversificam do da região formada pelas Zonas da Mata, metalúrgica, Vertentes e Belo Horizonte e arredores ${ }^{25}$. (1998, p. 32)

Toujours à propos des parlers caractéristiques de Minas Gerais, l'auteur synthétise ses arguments en démarquant et caractérisant trois parlers distincts dans l'État : le parler bahianais au nord, le parler paulista au sud et sud-est et le parler mineiro au centre-est. La carte $n^{0} 1^{26}$ permet de visualiser la distribution de ces parlers sur le territoire de Minas Gerais.

23. Trombe d'eau, n. f. : forte pluie, rapide mais accompagnée d'inondations.

24. «Relevé direct, in loco, dans différentes localités de Minas Gerais, précisément dans 184 points à travers un entretien semi-dirigé et des questionnaires spécifiques, conçus à cette fin.»

25. «L'État de Minas Gerais présente des accents, des phones, des rythmes de parole et des préférences lexicales distinctes dans au moins trois de ses régions, indépendamment de ses strates sociales. Il existe un parler dans le Sud et dans le Triangle qui se distingue du Nord, et qui se diversifient d'ailleurs des parlers de la région formée par Zonas da Mata, métallurgique, Vertentes et Belo Horizonte et alentours.»

26. Bien qu'il manque des éclaircissements à ce sujet, on peut supposer que cette carte devait apparaître sur l'un des volumes non publiés de l'EALMG, non seulement 


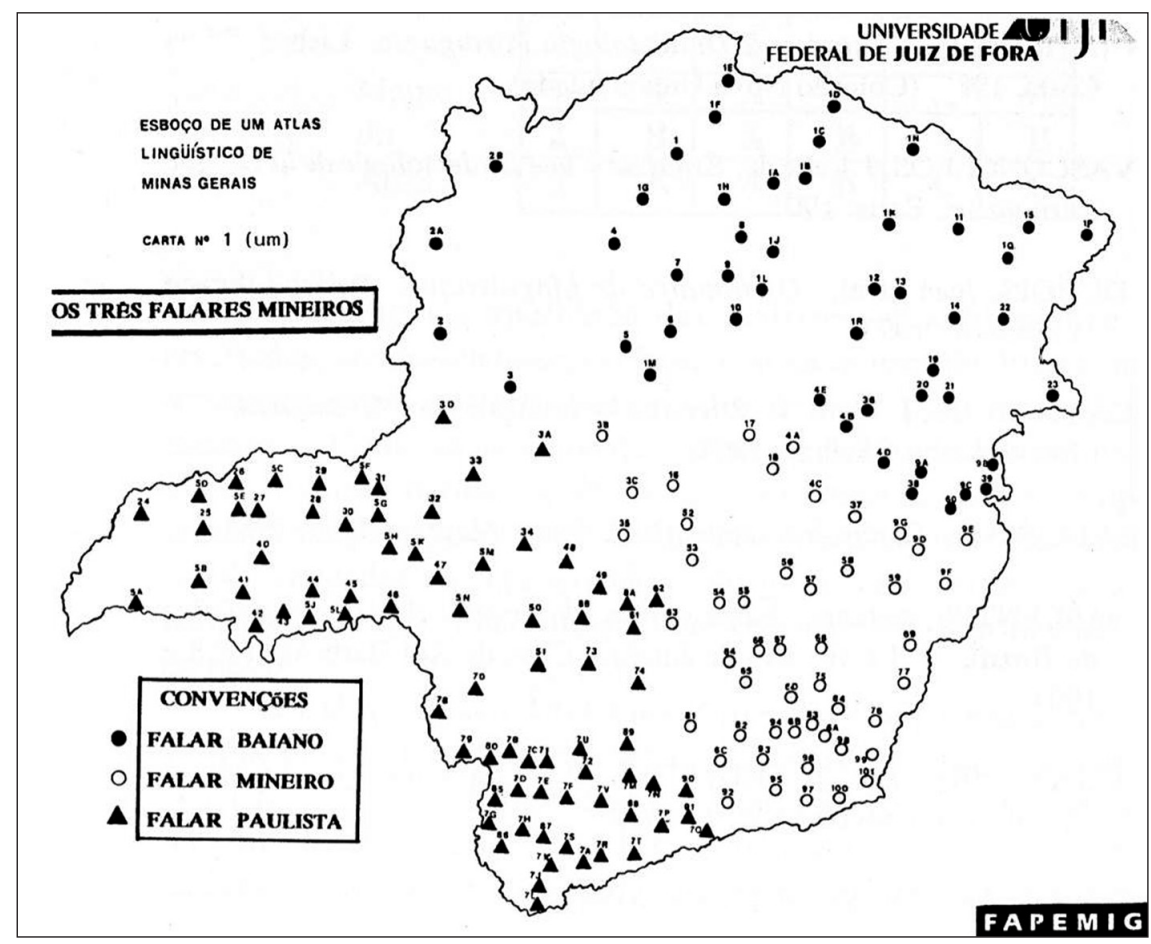

Figure 1. - Carte ${ }^{\circ} 1$ EALMG : les trois parlers de Minas Gerais.

Extrait de Zágari (1998, p. 46).

À la suite de ces commentaires sur l'EALMG, nous présentons les principales considérations à propos du deuxième atlas étudié dans le présent article, l'ALMS.

\subsection{Atlas Linguístico de Mato Grosso do Sul - ALMS : méthodologie}

Coordonné par Dercir Pedro de Oliveira, chercheur à l'Université fédérale de Mato Grosso do Sul (UFMS), l'ALMS a été publié en 2007 en un seul volume. Il représente le premier atlas linguistique consacré au parler de la région Centre-Ouest du Brésil. À la différence de l'EALMG, cet atlas a une dimension diatopique et contrôle les variables sociales, sexe et âge, ce qui le place parmi les atlas pluridimensionnels réalisés à partir des fondements de la dialectologie et de la géolinguistique contemporaines.

parce qu'elle présente une configuration graphique différente des autres cartes qui composent le volume I, mais aussi parce qu'elle introduit une nouvelle numérotation, comme il est possible de voir dans les autres cartes consultées (Zágari, 1998). 
Le projet de l'ALMS a été conçu et inauguré au début des années 1990 par Albana Xavier Nogueira, professeur chercheur attaché à la même université. En raison de son départ à la retraite en 1996, la coordination du projet a été assumée par Dercir Pedro de Oliveira jusqu'à sa dernière étape, c'est-à-dire la publication de l'atlas. L'équipe du projet était également formée par les chercheurs suivants, à différents moments de son exécution : Aparecida Negri Isquerdo, Ana Maria Pinto Pires de Oliveira, Maria José Toledo Gomes, Maria Leda Pinto et Vitória Regina Spanhero Ferreira. Le projet a été développé avec l'aide financière de la FUNDECT (Fondation de soutien au développement de l'enseignement, de la science et de la technologie de l'État de Mato Grosso do Sul) et du CNPq (Conseil national de développement scientifique et technologique).

\subsubsection{Le réseau de points}

L'ALMS recouvre un large réseau de points ${ }^{27}$, à savoir toutes les mésorégions et microrégions de l'État de Mato Grosso do Sul. Il est constitué de 32 points et comporte des villes de grande, moyenne et petite taille, en plus d'un district de la ville de Corumbá (intégrée au réseau dû à son importance historique à l'époque où elle était une station du chemin de fer Noroeste do Brasil, ce dernier reliant l'État de São Paulo à l'État de Mato Grosso do Sul) et des aires rurales du Pantanal (vaste zone marécageuse de Mato Grosso et Mato Grosso do Sul), un territoire couvert par trois points : Pantanal de Nhecolândia, de Nabileque et de Paiaguás. Lors du choix des localités, seules les municipalités créées jusqu'en 1980 ont été prises en compte, en vue de garantir la présence, dans la localité, d'informateurs adaptés au profil recherché. Les critères retenus lors de la sélection du réseau ont été les suivants : historique, frontalier et géométrique (Nogueira \& Isquerdo, 2005, p. 243). Les 32 localités du réseau de points sont

[...] distribuídas por cinco setore ${ }^{28}$, cujos municípios principais são: Três Lagoas, Corumbá, Aquidauana, Dourados e Campo Grande. A escolha das localidades teve como base aspectos demográficos, históricos e sociais ${ }^{29}$.

(Oliveira, 2007, p. 21)

27. À l'époque où le réseau de points de l'ALMS a été défini, l'État de Mato Grosso do Sul comptait 76 villes.

28. À l'origine, la structure du projet de l'ALMS comprenait cinq secteurs, choisis selon les différents sites de l'Université fédérale de Mato Grosso do Sul où travaillaient les chercheurs responsables de l'exécution du projet.

29. «[...] distribuées sur cinq secteurs, dont les villes principales sont : Três Lagoas, Corumbá, Aquidauana, Dourados et la capitale Campo Grande. Le choix des localités a suivi des critères démographiques, historiques et sociaux.» 
La section 8.3 (Réseau de points) de l'atlas contient la liste des localités, suivie de la présentation des informations historico-géographiques de 28 d'entre elles, parmi les 32 qui la composent, ce qui comprend : i) des données sur la fondation de la ville et son passage respectif au statut de district, municipalité et comarque, si pertinent; ii) le jour de son anniversaire; iii) son aire géographique et sa représentativité, en pourcentage, par rapport à l'ensemble du territoire de Mato Grosso do Sul; iv) la population de la municipalité, d'après l'IBGE (Institut brésilien de géographie et de statistique) de 2006.

\subsubsection{Le profil des informateurs}

Étant donné que l'ALMS était placé dans la catégorie des atlas pluridimensionnels, la méthodologie adoptée prévoyait le contrôle de la variable diatopique et des variables sociales. Selon ce paramètre,

Em cada localidade, foram selecionados quatro informantes, estratificados em sexo (masculino e feminino), grau de instrução (rudimentar ou com escolaridade até a $4^{a}$ série do ensino fundamental) e naturalidade (nascido no município ou nele residido desde os 8 anos de idade) ${ }^{30}$. (Oliveira, 2007, p. 16$)^{31}$

L'atlas fournit ensuite au lecteur un résumé sur les caractéristiques de chaque informateur : initiales du nom, état civil, profession, âge, scolarité et lieu de naissance. Parmi l'ensemble des informateurs, certains, notamment ceux qui se situaient dans la deuxième tranche d'âge, ne correspondaient pas au profil préétabli, fort probablement en raison de la difficulté de trouver des sujets présentant les caractéristiques retenues : dans sept localités, quelques informateurs avaient plus de 70 ans, et dans 23 autres ils n'étaient pas originaires de la localité, certains provenant d'autres villes de la même région linguistique ou d'autres unités de la fédération du Brésil. Les cas de non correspondance du profil de l'informateur quant au lieu de naissance peuvent être expliqués par l'histoire sociale de Mato Grosso do Sul, un État très marqué par les vagues migratoires.

30. «Dans chaque localité, quatre informateurs ont été sélectionnés selon le sexe (masculin et féminin), le degré d'instruction (rudimentaire ou scolarisé(e) jusqu'au CM2) et l'origine (né dans la ville ou y résidant depuis l'âge de 8 ans).»

31. Selon Nogueira et Isquerdo (2005, p. 245), les informateurs de l'ALMS ont été séparés en deux tranches d'âge : première tranche (18 à 30 ans); deuxième tranche (45 à 70 ans). Le recours à cette référence bibliographique se justifie par le fait que le texte de l'atlas ne contenait pas cette information. 


\subsubsection{Le questionnaire linguistique et les entretiens}

Le questionnaire de l'ALMS contient 557 questions, dont certaines contribuent à mettre en évidence des traits phonétiques et des aspects lexicaux (les questions concernent 14 champs sémantiques concernant la nature, l'homme, les superstitions/les rituels magiques, les légendes et, en plus une sollicitation pour obtenir des récits libres : «Racontez un fait de votre enfance que vous n'avez jamais oublié»). Ce dernier permettait en particulier l'étude de traits morphosyntaxiques : «O aspecto morfossintático foi estudado a partir das narrativas feitas pelos informantes, sobre fatos que tenham marcado suas vidas ${ }^{32}$.» (Oliveira, 2007, p. 20)

Selon Nogueira et Isquerdo «a elaboração do Questionário Linguístico do projeto ALMS pautou-se, sobretudo, no Questionário Linguístico do projeto do Atlas do Estado de São Paulo (1983), para a seleção das perguntas $^{33}{ }^{\prime}(2005$, p. 240). Les auteurs expliquent aussi quelles informations fournies par les Bases para a elaboração do Atlas Linguístico do Brasil de Nascentes $(1958,1961)$ ont également été prises en compte, ainsi que celles du Questionário Linguístico do projeto do Atlas Linguístico-Etnográfico de Portugal e Galiza (1974) et du Questionário do Atlas Linguístico do Ceará $(1982)^{34}$.

Le recueil des données a été réalisé par les chercheurs et par des étudiants d'initiation scientifique et de master, ces derniers dirigés par les professeurs faisant partie de l'équipe du projet. La méthode adoptée a été celle des entretiens, dans le cadre de la méthode directe, à l'aide du questionnaire unique : "Os inquéritos foram gravados 'in loco', com duração de mais ou menos três horas [...] totalizando trezentas e oitenta e quatro horas, com 128 informantes, sendo sessenta e quatro homens e sessenta e quatro mulheres ${ }^{35}$.» La transcription phonétique et graphématique des données et leur stockage respectif ont été faits à partir du logiciel SPDGL (Système de traitement de données géolinguistiques) (Oliveira, 2007, p. 20).

32. «L'aspect morphosyntaxique a été étudié à partir des récits des informateurs sur les faits qui avaient marqué leurs vies.»

33. «L'élaboration du questionnaire linguistique du projet ALMS s'est principalement basée sur le Questionário Linguístico do projeto do Atlas do Estado de São Paulo (1983).»

34. Ces informations sur les sources consultées pour l'élaboration du questionnaire ne sont pas fournies dans la section consacrée à la méthodologie, située dans le volume de l'ALMS.

35. «Les enquêtes ont été enregistrées in loco, avec une durée d'environ trois heures [...] totalisant trois cent quatre-vingt-quatre heures, avec 128 informateurs, dont soixante-quatre hommes et soixante-quatre femmes.» 


\subsubsection{L'organisation de l'Atlas Linguístico de Mato Grosso do Sul}

Le volume de l'ALMS s'ouvre sur une courte «Présentation» de l'ouvrage, signée par Fabiana Portela de Lima ${ }^{36}$. Suit une «Introduction», signée par l'éditeur de l'ouvrage, avec des commentaires généraux sur les études dialectologiques au Brésil : l'accent est mis sur la publication de l'Atlas Prévio dos Falares Baianos (Rossi, 1963), ainsi que sur la sortie du projet Atlas linguistique du Brésil (carte de Salvador) à la fin des années 1990 et l'interaction entre la dialectologie et la sociolinguistique dans les re-cherches dialectologiques contemporaines.

À la suite, le chapitre 1 «Origine» présente des données historiques du projet de l'ALMS, accompagnées du nom des chercheurs engagés dans l'élaboration de sa première version (Albana Xavier Nogueira et Maria José de Toledo Gomes); on y évoque également le changement de la coordination du projet, survenu à l'occasion du départ à la retraite de Albana Xavier Nogueira (1996), ainsi que l'aide financière attribuée au projet de la part de deux centres de financement à la recherche, appartenant à l'État (FUNDECT) et au gouvernement fédéral (CNPq). Le projet a été financé par le CNPq en partenariat avec l'Université fédérale de Rio de Janeiro (UFRJ). Cette institution a beaucoup contribué au projet dans le sens d'un «melhor tratamento científico dos dados [...] e discussões a respeito das interpretações dos dados e avaliações das cartas linguísticas elaboradas $^{37} \gg$ (Oliveira, 2007, p. 13).

Le volume de l'ALMS fournit par ailleurs les «Objectifs» de l'atlas (chapitre 2); la «Justification» de son élaboration (chapitre 3); la «Liste des chercheurs adjoints» (chapitre 4); la «Liste des enquêteurs» (chapitre 5); la «Liste des transcripteurs» (chapitre 6); le «Rapport enquêteurs / transcripteurs» (chapitre 7) ; les «Approches méthodologiques » (chapitre 8) divisées en deux sections : «Informateurs» (section 8.1), où l'on décrit le profil de ces derniers et où l'on fournit une liste des caractéristiques des quatre informateurs ${ }^{38}$ enquêtés dans les 32 localités qui forment le réseau. La section «Recueil des données» (section 8.2) contient pour sa part des informations générales sur la réalisation des enquêtes, la transcription et le stockage des données, alors que la section suivante, «Réseau de points» (section 8.3) fait le relevé des 32 localités qui forment le réseau de points, suivie de la caractéristion de chaque localité,

36. Membre de l'équipe des enquêteurs et transcripteurs (Oliveira, 2007, p. 15).

37. «meilleur traitement scientifique des données [...] et des discussions sur les interprétations des données et les évaluations des cartes linguistiques élaborées».

38. Dans la localité de Camapuã, on a enregistré les données d'un seul informateur. Voir la section 2.1.2 du présent article. 
comme décrit dans la section 2.1.1 du présent article. La section «Thèmes du questionnaire» (section 8.4) présente ensuite la liste des aires thématiques autour desquelles ont été rassemblées les questions qui composent l'instrument de la récolte des données de l'ALMS. Le chapitre «Système de symboles phonétiques» (chapitre 9) présente quant à lui la liste des symboles phonétiques employés lors de la confection des cartes linguistiques, dont la présentation apparaît au chapitre 10 «Cartes linguistiques», un thème que nous abordons par la suite.

L'ALMS réunit 217 cartes linguistiques ainsi distribuées : 57 cartes phonétiques; 153 cartes semantico-lexicales et 7 cartes morphosyntaxiques ${ }^{39}$. Selon Oliveira, «as cartas lingüísticas foram elaboradas por meio do programa computacional SPGDL (Sistema de Processamento de Dados Geolinguísticos $)^{40}$, e, posteriormente, editadas em programa de computação

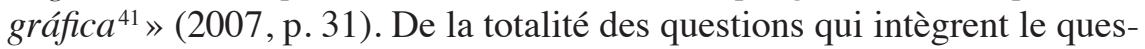
tionnaire linguistique de l'ALMS, un échantillon de thèmes a été sélectionné et «cartografaram-se somente as variantes lingüísticas mais identificadas com o Estado, que, na verdade, são resultantes, principalmente, de influências de mineiros, paulistas, gaúchos, índios, negros e pessoas das regiões fronteiriças ${ }^{42} \gg$ (Oliveira, 2007, p. 267).

La série de cartes de l'ALMS commence par la première carte phonétique; il n'y a donc pas de carte destinée à l'identification des éléments qui forment la base cartographique employée. Dans la configuration des cartes, une ligne marque la séparation entre l'espace où figure la base cartographique de l'ALMS, créée par le logiciel SPGDL, l'échelle de la carte et, à droite, la carte du Brésil en miniature où est mis en relief l'État de Mato Grosso do Sul : les informations spécifiques pour chaque carte sont données à droite de la page. Sur la partie supérieure figure le titre, différemment indiqué selon le type de carte. Dans les cartes phonétiques et lexico-sémantiques, ce dernier est précédé par le sigle du questionnaire d'où provient la question qui a donné origine à la carte (QFF : Questionnaire phonético-phonologique; QSL : Questionnaire sémantico-lexical),

39. Le volume de l'ALMS ne contient pas la liste des cartes présentées.

40. Le texte qui introduit la présentation des cartes (section 10) ne fournit pas d'informations sur les caractéristiques du logiciel SPGDL utilisé par l'équipe qui a produit l'ALMS, ni les critères adoptés pour la confection des cartes linguistiques.

41. «Les cartes linguistiques ont été élaborées à l'aide du logiciel SPGDL (Système de traitement de données géolinguistiques), éditées ensuite à travers un logiciel de cartographie.»

42. «On a cartographié seulement les variantes linguistiques les plus spécifiques à l'État, qui résultent en réalité et surtout de l'influence des Mineiros, Paulistas, Gauchos, Indiens, Noirs et des habitants des régions frontalières.» 
suivie du numéro de la question ${ }^{43}$ et de la lettre $a$ (lorsqu'il y a plus d'une carte portant sur le même phénomène, l'identification se fait par les lettres de l'alphabet $[\mathrm{b}, \mathrm{c} . .]$.$) . Quant aux cartes morphosyntaxiques, étant donné$ qu'elles ont été produites «a partir das narrativas feitas pelos informantes, sobre fatos que tenham marcado suas vidas ${ }^{44}{ }$ (Oliveira, 2007, p. 20), leur identification se fait à partir du nom relatif au type de carte, suivi du phénomène cartographié. Le tableau 1 ci-dessous illustre des titres des cartes de l'ALMS.

\begin{tabular}{|c|c|c|}
\hline Cartes phonétiques & Cartes lexico-sémantiques & Cartes morphosyntaxiques \\
\hline $\begin{array}{c}\text { QFF 01.a - FAMÍLIA } \\
\text { FAMÍ(LIA) } \\
\text { (fr. FAMILLE) }\end{array}$ & $\begin{array}{c}\text { QSL 0037.a - arco-iris } \\
\text { (fr. arc-en-ciel) }\end{array}$ & $\begin{array}{c}\text { CARTE } \\
\text { MORPHOSYNTAXIQUE } \\
\text { ON/NOUS }\end{array}$ \\
\hline $\begin{array}{c}\text { QFF 01.b - FAMÍLIA } \\
\text { (FA)MÍLIA }\end{array}$ & QSL 0037.b-arco-íris & $\begin{array}{c}\text { CARTE } \\
\text { MORPHOSYNTAXIQUE } \\
\text { PRONOM COMPLÉMENT }\end{array}$ \\
\hline $\begin{array}{c}\text { QFF 15.a - FERVENDO } \\
\text { FER(VENDO) } \\
\text { (fr. BOUILLANT) }\end{array}$ & $\begin{array}{c}\text { QSL 0090.a - joão-de-barro } \\
\text { (fr. fournier-roux) }\end{array}$ & $\begin{array}{c}\text { CARTE } \\
\text { MORPHOSYNTAXIQUE } \\
\text { PERTE DE L'OBJET }\end{array}$ \\
\hline $\begin{array}{c}\text { QFF 15.b - FERVENDO } \\
\text { (FER)VENDO }\end{array}$ & $\begin{array}{c}\text { QSL 0254.a - prostituta } \\
\text { (fr. prostituée) }\end{array}$ & $\begin{array}{c}\text { CARTE } \\
\text { MORPHOSYNTAXIQUE } \\
\text { AUXILIAIRE AVOIR }\end{array}$ \\
\hline
\end{tabular}

Tableau 1. - Exemples de titres des cartes de l'ALMS.

Élaboré par l'auteure à partir de l'ALMS.

L'équipe responsable de l'élaboration de l'atlas a choisi un système de symboles pour faciliter l'identification des désignations dans la carte-base. Dans les cartes phonétiques et morphosyntaxiques, ce système n'a pas compromis la lisibilité des données, les variantes étant en moindre quantité. Néanmoins, dans les cartes lexico-sémantiques, étant donné que le critère de présentation retenu n'a pas été celui de l'item lexical orthographié selon la norme du portugais, mais la variante phonétique de la forme fournie comme réponse, il a été nécessaire de créer une quantité assez grande de symboles pour indiquer les variantes d'une même unité lexicale. À titre d'exemple, dans la carte QSL 0132.a - moustique, pour l'item lexical per-

43. Comme le Questionnaire linguistique utilisé pour le recueil des données n'est pas présenté dans la section correspondante à ce thème, ainsi que le concept objet de la question qui a donné origine aux données cartographiées, ce critère d'identification de la carte rend difficile l'interprétation de la part du lecteur de l'atlas.

44. «à partir des récits des informateurs sur des faits ayant marqué leurs vies». 
nilongo ${ }^{45}$, apparaissent les variantes phonétiques suivantes, recupérables par un symbole spécifique : pernilongu, pernelongu, penelongu, pernilongu, pirnilongu, pernolingu et pernalongu; et pour moustique, la carte présente les formes musquitu et mosquitu. Sur les légendes figurent aussi les sigles RP et RNP : étant donné que ces derniers n'ont pas été définis dans la méthodologie de l'atlas, on suppose qu'ils indiquent, respectivement, réponses annulées (RP) et réponses non demandées (RNP).

Quant à l'identification des informateurs dans les cartes linguistiques, le système mis en place est celui de la croix $(+)$, largement diffusé dans la présentation des données linguistiques des cartes des atlas aux traits pluridimensionnels, à l'exemple de l'ALMS. La codification adoptée est présentée dans la partie inférieure de la colonne destinée à l'identification des données contenues dans les cartes. Sur la ligne horizontale de la croix figurent les informations d'ordre diagénérationnel - au-dessus de la ligne se trouvent les informateurs appartenant à la tranche d'âge I et, au-dessous, ceux de la tranche d'âge II - , alors que la ligne verticale distingue les informateurs selon la dimension sexuelle - à gauche, se situent les informateurs du sexe masculin, et à droite ceux du sexe féminin. Dans la carte-base, le symbole correspondant à chaque variante est identifié par des couleurs différentes, qui indiquent l'informateur : vert (homme appartenant à la première tranche d'âge), blanc - absence de couleur - (femme appartenant à la première tranche d'âge), bleu clair (homme appartenant à la deuxième tranche d'âge, jaune (femme appartenant à la deuxième tranche d'âge). Le fond de la carte-base étant en vert clair, cette duplication des critères dans l'identification de l'informateur (position dans l'image de la croix et couleur), à laquelle s'ajoute la grande quantité de symboles utilisés pour indiquer les diverses désignations, en particulier dans les cartes lexico-sémantiques, sont des facteurs qui compromettent largement la lecture des données.

Pour terminer cette description de la nature des cartes linguistiques de l'ALMS, nous tenons à en signaler deux aspects positifs. En premier lieu, la valorisation de la réalité régionale, rurale, dans le choix des questions du questionnaire qui ont fait l'objet de la cartographie, par les aspects suivants $^{46}$ : natureza - QSL 0005.a - banhado/corixo; QSL 0011.a - vau;

45. Variété de moustique.

46. Par ordre d'apparition : nature; canal d'eau qui relie les baies dans la région du Pantanal; gué; marée descendante; baie; animaux; se dit d'un animal qui n'a pas d'oreilles, ou qui n'en a qu'une; se dit d'un cheval qui a une hanche plus élevée que l'autre; selle; se dit du veau qui a plus d'un an; taureau sauvage du Pantanal; croyances régionales; cortège de la croix; cercle de la lune; habitudes alimentaires; ragoût de viande et manioc; riz préparé avec de la viande séchée; gâteau salé au 
0013.a - vazante; QSL 015.a - baía; animais - QSL 0098.a - nambi; QSL 0099.a - lonanco; QSL 0104.a - baldana; QSL 0109.a - sobreano; QSL 0112.a - bagual; crenças regionais - QSL 0309.a - velório da cruz; QSL 0029.b - círculo da lua; hábitos alimentares - QSL 0340.a caribéu; QSL 0342.a - arroz carreteiro; QSL 0343.a - sopa paraguaia; QSL 0352.a-tereré; QSL 0354.a - matula.

En deuxième lieu, l'insertion des données d'ordre morphosyntaxique dans la cartographie des données, alors que l'instrument de recueil ne prévoyait pas de questionnaire spécifique avec, notamment, des questions conçues pour ce niveau de description linguistique. Le relevé et le contrôle des phénomènes ont ainsi été réalisés à partir des textes motivés par la proposition du récit spontané.

Dans la suite du volume de l'atlas, le chapitre 11 «Considérations sur le lexique », signé par Vitória Regina Spanghero Ferreira ${ }^{47}$, présente une étude sur un découpage du lexique cartographié dans les 153 cartes lexicosémantiques (29 unités lexicales). À partir de la consultation de quelques dictionnaires de la langue portugaise, l'auteure informe l'étymologie de la variante, la datation ${ }^{48}$, la définition de l'item lexical, les variantes de l'item lexical en question, ses marques d'usage lorsque les variantes sont enregistrées dans les ouvrages lexicographiques consultés, ainsi que les dénominations considérées comme appartenant au vocabulaire de l'État de Mato Grosso do Sul, c'est-à-dire des variantes lexicales cartographiées et recueillies à partir du vocabulaire des sujets parlants enquêtés, puis documentées dans l'ALMS.

\section{En voici un exemple : Nambi}

Etimologia: do tupi nambí "orelha".

Acepções: substantivo masculino. Orelha animal ou humana. Adjetivo de dois gêneros. Que não tem orelhas ou tem apenas uma. Que tem uma ou mais as duas orelhas caídas (cavalo). Que não tem rabo.

Denominações sul-mato-grossenses: troncho, burro, cabano, mocho, nambi, banana, troncha, gancho, tronchinho, manco, báiu, tronco, troxu ${ }^{49}$. (Ferreira, 2007, p. 255)

maïs, plat typique du Paraguay; infusion d'herbe de maté, boisson froide typique de l'État; casse-croûte.

47. Membre de l'équipe qui a produit l'ALMS : chercheur adjoint.

48. Ces informations n'apparaissent pas dans toutes les unités lexicales analysées.

49. Nambi. Étymologie : du tupi na'mbi : oreille. Acceptions : nom féminin. Oreille animale ou humaine. Adjectif de deux genres. Qui n'a pas d'oreilles ou n'en a qu'une. Qui a une oreille, ou les deux, pendue(s) (cheval). Qui n'a pas de queue. Dénominations issues du vocabulaire de Mato Grosso do Sul : troncho, burro, cabano, mocho, nambi, banana, troncha, gancho, tronchinho, manco, báiu, tronco, troxu. 
L'échantillon du lexique dialectal réuni au chapitre 11 de l'ALMS illustre à la perfection la richesse lexicale pérénisée par l'atlas, dans la mesure où il représente

[...] uma porção do léxico sul-mato-grossense, com exemplos de nomes de animais, plantas, fenômenos naturais, alimentos, partes do corpo, entre outras palavras que demonstram a visão de mundo dos falantes dessa região ${ }^{50}$. (Ferreira, 2007, p. 266)

L'ouvrage se termine par la «Conclusion», les «Références» et un recueil de photos qui documentent des scènes de l'équipe de recherche de l'ALMS dans différentes localités du réseau de points et des paysages naturels de différentes régions de l'État de Mato Grosso do Sul.

\section{L'arc-en-ciel dans I'EALMG et l'ATELMS}

Comme nous l'avons signalé dans l'introduction du présent article, les deux atlas étudiés documentent la langue portugaise parlée dans les unités de la fédération appartenant à deux grandes régions du Brésil, la région Sud-Est (EALMG) et la région Centre-Ouest (ALMS). L'histoire sociale du Brésil s'est déroulée sous le signe de la migration, tout particulièrement les aires territoriales qui n'ont été effectivement peuplées que récemment, comme c'est le cas pour les régions Centre-Ouest et Nord ( $\mathrm{XIX}^{\mathrm{e}}$ et $\mathrm{xx}^{\mathrm{e}}$ siècles). L'histoire de l'État de Mato Grosso do Sul, par exemple, témoigne de la forte présence des Mineiros au tout début de la période de peuplement de diverses régions de l’État. Sa capitale, Campo Grande, et Dourados, sa deuxième plus grande ville, ont été fondées par les Mineiros lorsque ces derniers quittèrent Minas Gerais pour travailler dans les fermes d'élevage bovin, dans les prairies assez propices à l'agriculture et à l'élevage lui-même. La partie située à l'est de Mato Grosso do Sul fait frontière avec la région du Triângulo Mineiro, l'aire du parler paulista, selon Zágari (1998), d'après la carte identifiée dans la figure 1 de notre article.

Dans ce cadre historique, nous examinons, dans la présente section, des cartes lexicales présentes dans les deux atlas en question, autour du même thème : le phénomène atmosphérique qui consiste en la présence d'une bande lumineuse aux rayons colorés et courbés qui peuvent apparaître dans le ciel à la suite d'une pluie, communément désigné arc-en-ciel.

50. «[...] une partie du lexique de Mato Grosso do Sul, avec des exemples de noms d'animaux, de plantes, de phénomènes naturels, d'aliments, de parties du corps, parmi d'autres mots qui témoignent de la vision de monde des sujets parlants de cette région.» 
De l'EALMG, nous avons pris pour source de données la carte $\mathrm{n}^{\circ} 1 \mathrm{ARCO}-$ ÍRIS et, de l'ALMS, les cartes QSL 0037.a - arco-íris et QSL 0037.b arco-íris. En faisant un parallèle entre les désignations extraites de ces cartes, on observe aussi bien la distribution spatiale des données que la proportion dans laquelle des unités lexicales se sont diffusées et figées dans le lexique des Mineiros et des habitants de Mato Grosso do Sul, en prenant pour référence l'histoire sociale des deux régions brésiliennes représentées par les atlas. Ceci dit, les étapes parcourues ont été les suivantes : a) relevé des désignations pour arco-íris dans les cartes sélectionnées; b) analyse diatopique des unités lexicales identifiées; c) considérations sur la motivation des variantes : perspective linguistique et anthropologique.

Le tableau 2, ci-dessous, présente le corpus analysé, distribué selon la source consultée. Les numéros placés dans les colonnes indiquent la quantité de localités du réseau de l'atlas où la désignation a été documentée, en considérant cependant le fait que le réseau de l'EALMG comportait 116 localités, alors que celui de l'ALMS en avait seulement 32 .

\begin{tabular}{|c|c|c|}
\hline Variante lexicale & $\begin{array}{c}\text { EALMG } \\
{\text { Carte }{ }^{\circ} 1 \text { ARCO-ÍRIS }}^{\circ} \text { ARC }\end{array}$ & $\begin{array}{c}\text { ALMS } \\
\text { Cartes QSL 0037.a / } \\
\text { QSL 0037.b-arco-íris }\end{array}$ \\
\hline Arco & & 1 \\
\hline Arco-íris & 89 & 32 \\
\hline Arco celeste & & 1 \\
\hline Arco-da-velha/Arco-do-velho & 57 & 1 \\
\hline Arco-do-sol & 3 & \\
\hline Arco-da-aliança & 3 & \\
\hline Arco-de-Noé & & 1 \\
\hline Aliança & & 1 \\
\hline Aliança-de-Deus & & 2 \\
\hline Rabo de Galo & 5 & 2 \\
\hline Rabo-de-pavão & 1 & \\
\hline Olho-de-boi & 1 & \\
\hline Mãe d'Água & 1 & \\
\hline Navio & 1 & \\
\hline Barrado & & 1 \\
\hline Círculo & & 1 \\
\hline Ocurismo & & 1 \\
\hline Papa peixe & & 1 \\
\hline
\end{tabular}

Tableau 2. - Désignations pour arco-íris dans I'EALMG et l'ALMS.

Élaboré par l'auteure à partir de l'EALMG et l'ALMS ${ }^{51}$.

51. Par ordre d'apparition dans le tableau : arc, arc-en-ciel, arc céleste, arc de la vieille/ arc du vieux, arc du soleil, arc de l'alliance, arc de Noé, alliance, alliance de Dieu, 
Les données du tableau 2 montrent que les unités lexicales arco-íris et arco-da-velha ont été documentées dans un plus grand nombre de localités du réseau de l'EALMG, alors que, dans celui de l'ALMS, la forme arco-íris est privilégiée, la désignation arco-da-velha ayant été relevée dans seulement une localité de l'État de Mato Grosso do Sul - la ville de Camapuã (informateur féminin jeune).

Sont également représentées comme des variantes communes, dans les deux atlas, des désignations constituées par l'élément aliança : arco-daaliança dans l'EALMG, relevées dans trois localités, l'une au centre, dans une aire considérée comme relevant du parler mineiro, et deux au sud de Minas Gerais, dans une aire du parler paulista selon Zágari (1998) (fig. 1), ainsi que aliança et aliança-de-Deus, documentées par l'ALMS, respectivement, dans une et dans deux localités. La variante aliança a été mentionnée par l'informateur masculin de la tranche d'âge II, originaire d'Inocência, une ville de la mésorégion située à l'est de l'État qui fait frontière avec celui de Minas Gerais, aire du parler paulista. Aliança-de-Deus, tout comme la variante précédente, a également été mentionnée par les informateurs masculins de la deuxième tranche d'âge, dans les villes de Bonito et Pedro Gomes, situées respectivement dans les mésorégions Sud-Ouest et CentreOuest de l'État.

Une autre désignation relevée dans les deux atlas est rabo-de-galo, avec un total de cinq localités dans l'EALMG et deux localités dans l'ALMS. À Minas Gerais, rabo-de-galo s'est répandue dans les aires des trois types de parlers mentionnés par Zágari (1998) : l'une sur la zone territoriale du parler bahianais (État de Bahia) et sur celle du parler paulista (État de São Paulo), et trois dans l'aire du parler mineiro. Dans l'État de Mato Grosso do Sul, la variante rabo-de-galo a été relevée dans le Pantanal de Nhecolândia (aire rurale de la ville de Corumbá, mésorégion du Pantantal de Mato Grosso do Sul) et à Sete Quedas (ville du sud de l'État, mésorégion Sud-Ouest qui fait frontière avec le Paraguay), par des informateurs de la tranche d'âge II, appartenant respectivement au sexe masculin et féminin. L'une des variantes n'a été relevée qu'à Minas Gerais, dans trois localités de l'aire du parler paulista. Il s'agit de arco-do-sol. Contenant elles aussi la forme arco, dans l'ALMS apparaissent les désignations arcoceleste et arco-de-Noé, toutes deux en occurrence unique, documentées respectivement à Porto Esperança ${ }^{52}$ et Àgua Clara. Dans ces deux localités,

queue de coq, queue de paon, œil de bœuf, mère des eaux, navire, rayure, cercle, ocurismo, papa peixe.

52. Petit district de la municipalité de Corumbá, localisé sur les rives de la rivière Paraguay, dont l'accès se fait par voie fluviale. 
les variantes ont été mentionnées par l'informateur femme de la tranche d'âge II. Les données révèlent aussi d'autres cas d'occurrences uniques dans les deux atlas : quatre dans L'EALMG (olho-de-boi, mãe-d'água, rabo-de-pavão et navio) et cinq dans l'ALMS (círculo, barrado, arco-deNoé, ocurismo et papa peixe).

En ce qui concerne la motivation des désignations, la présence de l'unité lexicale círculo, pour nommer le phénomène optique couramment désigné arco-íris, semble provenir de la nature similaire des phénomènes nommés. L'ALMS présente deux cartes consacrées au phénomène atmosphérique círculo-da-lua ${ }^{53}$, c'est-à-dire le halo lunaire, une espèce d'anneau de lumière apparaissant autour de la lune, à la pleine lune, un effet provoqué par la pénétration de la lumière lunaire dans la troposphère. Il s'agit d'un phénomène formé par un processus semblable à celui de l'arc-en-ciel, à la différence que ce dernier est provoqué par la pénétration de la lumière solaire dans l'atmosphère. Dans l'imaginaire populaire de l'homme rural, círculo-da-lua indique un changement climatique : le cercle proche indique la pluie au loin; le cercle au loin indique l'approche de la pluie. La carte QSL 0029.a - círculo na lua ${ }^{54}$ atteste la présence de deux occurrences de la variante arco-íris (villes d'Eldorado et Porto Esperança) et de cinq occurrences de l'item lexical arco (Porto Esperança, Três Lagoas, Nioaque, Fátima do Sul et Amambaî) pour désigner le phénomène dit círculo na lua. Ces localités sont distribuées dans quatre des cinq mésorégions de l'État de Mato Grosso do Sul.

Quant à barrado ${ }^{55}$, en tant que variante lexicale de arco-íris dans la ville de Bandeirantes (homme de la tranche d'âge II), région centrale de l'État, son emploi pourrait s'expliquer par l'association du phénomène optique de l'arc-en-ciel avec un autre, relevant de la lumière solaire au lever du soleil : l'aurore, qui en milieu rural reçoit en général la désignation barra do dia ${ }^{56}$. Il s'agit d'un nom très usuel dans les chansons populaires, en particulier celles dont la thématique tourne autour des éléments du monde rural.

Dans une étude sur les désignations du phénomène de l'arc-en-ciel dans cinq atlas régionaux (parmi lesquels l'EALMG), Ferreira et Cardoso (1999,

53. La carte QSL 0029.a - cercle dans la lune - présente les désignations relevées pour nommer ce phénomène, et la carte QSL 0029.b - cercle de la lune - a relevé différentes significations qui lui sont attribuées dans l'imaginaire populaire du natif de Mato Grosso do Sul.

54. Cercle dans la lune.

55. Rayure, bande.

56. Rayure de la journée. Ferreira et Cardoso (1999) signalent l'usage de barra (rayure, bande) pour désigner le même phénomène à Sergipe et à Bahia. 
p. 23) proposent trois groupes pour classer des formes employées pour désigner ce phénomène. Elles sont formées à partir de l'élément arco, basé sur le deuxième élément de la forme composée. Si l'on transpose cette démarche aux données qui font l'objet de notre étude, nous avons la distribution suivante : i) référence aux animaux : $\operatorname{arco}-d e-b o i^{57}$; ii) terme religieux : arco-da-aliança, arco-de-Noé.

D'autres désignations avec l'élément arco ont été relevées dans les deux atlas, renvoyant à la sphère mythologique : arco-da-velha et arco-do-velho. Dans ses considérations sur les cartes de l'arc-en-ciel dans l'ALE (Atlas Linguarium Europae), Alinei analyse ces désignations et les classe en tant qu'anthropomorphisme païen, car il envisage l'unité lexicale vieux/vieille d'un point de vue mythologique : «la vieille, sorcière, femme sage, vieille boiteuse $^{58} \gg(1983$, p. 70), une interprétation que nous partageons. Dans cette catégorie s'insère aussi la variante arco-íris, si l'on considère que le deuxième élément de la forme composée a été motivée par la «divindade Iris, mensageira alada dos deuses que se recobria com um xale de sete cores identificado com o próprio arco-íri ${ }^{59} \gg$ (Ferreira \& Cardoso, 1999, p. 24).

Alinei considère que «the motivation 'bow' neither involves nor excludes qualifications of a mythological nature: we can have either an impessoal 'rainbow' or a mythological 'God's bow'» (1983, p. 48). En se fiant à ce présupposé et à l'analyse des vastes archives de désignations pour le phénomène de l'arc-en-ciel disponibles dans plusieurs pays européens, faisant donc l'objet de différentes cultures, Alinei réunit en trois groupes les désignations soumises à l'analyse : «from an ancient zoomorphic conception, through an early, pagan, anthropomorphic stage, to a later, Christian or Islamic, anthropomorphic transformations» (ibid., p. 50). De ce point de vue, arco-da-aliança se situerait dans la catégorie des anthropomorphismes chrétiens. Parmi les données fournies par l'ALMS, appartiennent également à cette cause dénominative les variantes aliança, aliança-deDeus et arco-de-Noé, lesquelles renvoient elles aussi aux fondements de la Bible chrétienne (ibid., p. 74).

57. Arc de bœuf.

58. À propos de cette interprétation, Ferreira et Cardoso (1999) se posent la question suivante : «Arco-da-velha et par conséquent arco-do-velho ne relèveraient-ils pas d'un cas d'anthropomorphisme chrétien, un raccourci de l'arc de la vieille alliance?» C'est une interprétation qui mérite d'être prise en compte dans la recherche.

59. «la divinité Iris, messagère ailée des dieux, qui se recouvrait d'une écharpe aux sept couleurs, identifiée à l'arc-en-ciel lui-même» . 
Par ailleurs, le chercheur italien se réfère au caractère ancien de la pratique de nomination du phénomène de l'arc-en-ciel motivée par les animaux : "The most archaic level is this reconstruction is represented by those names for the rainbow that are motivated by an 'animal', and thus reflect an ancient totemic vision of reality.» (Ibid., p. 50)

Les données que nous présentons révèlent la conservation de la référence aux animaux dans la manière de nommer le phénomène de l'arcen-ciel : rabo-de-galo (EALMG et ALMS); rabo-de-pavão, olho-de-boi (EALMG) et papa-peixe (ALMS). Dans l'analyse des désignations formées avec des noms d'animaux - zoomorphismes - Alinei fait mention au mythe, présent dans le folklore de presque tout le continent européen, selon lequel «the rainbow is a gigantic animal-most often a snake-that 'drinks' or 'sucks' water, as well as people and animals, from the earth and eventually spits them out» $(1983$, p. 50). La culture ibérique apportée par les colonisateurs des Amériques s'est répandue et plusieurs mythes ont été reconstruits et matérialisés à travers les désignations relevant des phénomènes de la nature, comme c'est le cas pour arc-en-ciel. Ce qui vient d'être affirmé se confirme par la présence de certains items lexicaux, comme arco-da-velha, arco-celeste, arco-da-aliança, arco-de-Noé, olho-de-boi, documentés dans différentes régions de l'Europe, selon les données fournies par l'Atlas Linguarum Europae - ALE (Alinei, 1983).

\section{Considérations finales}

Dans le but de faire des considérations sur deux atlas brésiliens, ce travail s'est principalement centré sur les paramètres méthodologiques adoptés pour leur production. Comme nous l'avons indiqué précédemment, trente ans séparent l'élaboration de ces deux atlas, qui ont, par la suite, documenté deux moments distincts de l'histoire du portugais brésilien, au-delà de la réalité linguistique régionale, étant donné qu'ils recensent des données linguistiques de deux États appartenant, respectivement, à la région Sud-Est et Centre-Ouest du Brésil. Alors que l'EALMG prend pour paramètres la dialectologie traditionnelle centrée sur la diatopie, l'ALMS s'est approprié les orientations des tendances contemporaines de la dialectologie, laquelle prend en compte la dimension spatiale mais aussi sociale, en l'occurrence, diasexuelle et diagénérationnelle. Il s'agit donc d'un atlas de type pluridimensionnel.

La mise en parallèle entre les données documentées dans les cartes autour du thème de l'arc-en-ciel, dans les deux atlas régionaux, témoigne de la diffusion d'items lexicaux comme arco-da-velha et rabo-de-galo par les Mineiros qui ont peuplé une grande partie du territoire de Mato Grosso 
do Sul. Dans l'EALMG, l'on remarque la concurrence entre l'unité lexicale arco-íris, dont l'usage est répandu dans la langue, et la forme plus régionale arco-da-velha. Quant aux données de l'ALMS, elles indiquent l'emploi systématique de arco-íris par les habitants de Mato Grosso do Sul, ainsi que la présence de variantes régionales typiquement rurales dans des aires précises, notamment celles qui ont été moins exposées aux influences de la société moderne, comme le Pantanal de Mato Grosso do Sul, ou les localités proches des limites nationales et internationales.

Toujours à propos de la date de publication des atlas, les études faites à partir des données fournies par ces ouvrages méritent d'être soulignées. Dans le cas de l'EALMG, les recherches sur les données cartographiées, enrichies par les résultats de travaux postérieurs à la publication - dont le but était de donner des subsides à la publication des autres volumes prévus dans le projet original -, indiquent la délimitation d'aires dialectales dans la langue portugaise parlée à Minas Gerais (voir la fig. 1). Quant aux études sur les données de l'ALMS, celles-ci ne sont pas encore suffisantes pour la délimitation d'aires dialectales du parler caractéristique de Mato Grosso do Sul.

En guise de conclusion, il est pertinent de rendre ici un hommage posthume aux chercheurs brésiliens dont les travaux ont abouti à la publication des deux atlas présentés, en reconnaissance à leur héritage pour les recherches dialectales et géolinguistiques au Brésil : José Ribeiro et Mário Roberto Lobuglio Zágari (deux auteurs de l'EALMG) et Dercir Pedro de Oliveira (éditeur de l'ALMS).

\section{RÉFÉRENCES BIBLIOGRAPHIQUES}

Alinei Mario, 1983, «Arc-en-ciel, ALE I, 1, Commentaires », dans M. Alinei et al., Atlas Linguarum Europae (ALE), t. I, Assen-Maastricht, Van Gorcum, p. 47-80.

Ferreira Carlota da Silveira \& CARdoso Suzana Alice Marcelino, 1999, «Arcoíris no Brasil: um estudo lingüístico-antropológico a partir dos atlas regionais», Revista do GELNE, vol. 1, n 2, p. 21-24. Disponible sur <www.gelne. org.br> [dernier accès le 30 mars 2015].

FERREIRA Vitória Regina Spanghero, 2007, «Considerações sobre o léxico », dans D.-P. Oliveira (éd.), Atlas Lingüístico de Mato Grosso do Sul, Campo Grande (MS), UFMS, p. 251-266.

MotA Jacyra Andrade \& CARDoso Suzana Alice Marcelino, 2006, «Para uma nova divisão dos estudos dialetais brasileiros», dans J.-A. Mota et S. A. M. Cardoso (éds), Documentos 2: Projeto Atlas Linguístico do Brasil, Salvador, Quarteto Editora, p. 15-26. 
Nogueira Albana Xavier \& Isquerdo Aparecida Negri, 2005, «Atlas Lingüístico de Mato Grosso do Sul: gênese e trajetória», dans V. d. A. Aguilera (éd.), A Geolinguística no Brasil: trilhas seguidas, caminhos a percorrer, Londrina, EDUEL, p. 229-250.

Oliveira Dercir Pedro (éd.), 2007, Atlas Lingüístico de Mato Grosso do Sul, Campo Grande (MS), UFMS.

Ribeiro José, Zàgari Mário Roberto Lobuglio, Passini José \& Gaio Antônio, 1977, Esboço de um Atlas Lingüístico de Minas Gerais, vol. 1, Rio de Janeiro, Fundação Casa de Rui Barbosa; Juiz de Fora, Universidade Federal de Juiz de Fora.

Rocha Ana Paula A., 2012, «Notas sobre o léxico de brincadeiras infantis usado em Minas Gerais à luz de dois trabalhos geolinguísticos: o ALEMiG (1977) e o Projeto ALiB », dans F.-C. Altino (éd.), Múltiplos olhares sobre a diversidade linguística: uma homenagem a Vanderci de Andrade Aguilera, Londrina, Midiograf, p. 79-92.

ZÀGARI Mário Roberto Lobuglio, 1998, «Os falares mineiros: esboço de um Atlas Linguístico de Minas Gerais», dans V. d. A. Aguilera (éd.), A Geolinguística no Brasil: caminhos e perspectivas, Londrina, EDUEL, p. 31-54.

Thun Harald, 1998, «La géographie linguistique romane à la fin du Xx siècle », dans Vivacité et diversité de la variation linguistique, actes du Congrès international de linguistique et de philologie romanes (Bruxelles, 1998), Tübingen, Niemeyer, vol. 3, p. 367-386. 